\title{
Autoimmunity, T-cells and STAT-4 in the pathogenesis of chronic obstructive pulmonary disease
}

\author{
M.G. Cosio
}

There is not much doubt that cigarette smoking is the main trigger for the development of chronic obstructive pulmonary disease (COPD). However, the mechanisms by which cigarettes produce the disease have been elusive. The consideration of new ideas could bring new insights. The paper by DI STEFANO et al. [1] in this issue of the European Respiratory Journal invites me to revisit a previously stated hypothesis [2-4], that the T-cell and autoimmunity may be the key in the mechanisms leading to COPD, since DI STEFANO et al. [1] provide another piece of evidence in favour of this paradigm.

Let's look at the autoimmune hypothesis with open eyes. Concepts evolve as new evidence settles in. Who would have believed 20 or even 10 yrs ago that peptic ulcer is an infectious disease, now treated with antibiotics, or that arteriosclerosis is an autoimmune disease, triggered by epitopes generated in the process of atherogenesis, such as oxidised low-density lipoproteins [5, 6]? Therefore, why not consider COPD as an autoimmune disease triggered by smoking? But why should we consider such as scenario? I will explain and, in doing so, how the Di STEFANo et al. [1] paper fits into this paradigm.

For years, the inflammatory reaction in COPD was focused on neutrophils and their elastase, and macrophages and their metalloproteinases: the protease-antiprotease paradigm; an important mechanism, but probably only one step in the complex pathogenesis of COPD. Then, FINKELSTEIN et al. [7] introduced the T-cell as an important inflammatory component in the lungs of smokers, expanding the field of investigation of the inflammatory reaction in COPD to include the T-cells. For years, it was believed that because neutrophils and macrophages were increased in smokers, they were involved in the pathogenesis of the disease. T-cells are also increased in smokers' lungs, thus it is necessary to determine why and what the consequences of this increase are.

To start, let's remember that naïve, nonstimulated T-cells cannot enter the lung, or any organ for that matter, because their homing receptors make them traffic to the lymphatic tissue [8]. Thus, the cells found in the lungs of smokers with COPD have to be primed and stimulated in order to get there. How could they be stimulated?

The concept of inflammation has evolved substantially since the findings of neutrophils and macrophages in the bronchoalveolar lavage (BAL) of smokers $[9,10]$. It is now recognised that the inflammatory reaction composed of neutrophils and macrophages represents the innate immune reaction, and that the adaptive immune response involving B- and T-cells is intimately linked with innate immunity. Therefore, the traditional inflammation in smokers (neutrophils and macrophages) should be interpreted as the innate immune response to cigarettes, which could lead to an adaptive response with T-cells.

Correspondence: M.G. Cosio, McGill University, Royal Victoria Hospital, 687 Pine Avenue West, Montreal, Quebec, H3A 1A1, Canada. Fax: 1 5148431695. E-mail: manuel.cosio@muhc.mcgill.ca
The function of the immune system (inflammation) was probably devised to defend against infectious microbes; however, even noninfectious foreign substances can elicit immune responses. This is because the receptors originally devised to recognise invading pathogens, the Toll receptors, also recognise "danger signals" from injured tissues (necrotic and apoptotic cells, proteins and peptides) regardless of the trigger: the "danger hypothesis" of MATZINGER [11]. Therefore, a more inclusive definition of immunity is a reaction to foreign substances including microbes, as well as to macromolecules such as proteins and polysaccharides and other agents, e.g. cigarette smoke, regardless of the physiological or pathological consequences of such a reaction [12]. Unfortunately, mechanisms that normally protect individuals from infection and eliminate foreign substances are themselves capable of causing tissue injury and disease in some situations, e.g. rheumatoid arthritis or COPD.

The present evidence suggests that the epithelium, by sending "danger" signals in response to cigarette smoke, is responsible for the initiation and possibly the maintenance of the innate immune response seen in smokers [13]. Once established, the innate inflammatory reaction, along with $>2,000$ xenobiotics and $10^{14}$ free radicals in each puff of cigarette smoke, will proceed over time to damage the lung, producing peptides and modified proteins (e.g. desmosine) from matrix destruction, cell necrosis and cell apoptosis. These products do not go unnoticed, since they have the potential to act as antigenic determinants, thus reaching the abundant dendritic cells (DCs) and other antigen presenting cells (APCs) that accompany the innate immune inflammation in smokers. In support of this possibility is the extensive evidence in the literature, which is easily applicable to cigarette smoking, indicating that infectious and environmental agents causing tissue damage have great potential for releasing and/or altering self-proteins, which could then be recognised as antigens by the adaptive immune system $[14,15]$.

DCs, which are abundantly present in smokers' lungs, are the key cellular links between innate and adaptive immunity. They play a pivotal role as sensors of infection or, in this case, injury for the initiation of an adaptive immune response involving T-cells. Here again, the innate immune response (neutrophils and macrophages among other cells) will be determinant of the progression from innate to adaptive immunity involving the T-cells. Not only is the innate inflammation producing potential antigens, but it also provides the mediators, chemokines, cytokines and costimulators that are necessary in the DC microenvironment for these cells to mature and migrate to the draining lymphatic organs, where they could present antigens to CD4+ and CD-8+ T-cells that induce their activation and differentiation.

A recently proposed model for $\mathrm{T}$-cell activation and differentiation [16], which fits nicely with the findings of DI STEFANO et al. [1], envisions the following scenario. After T-cell receptor (TCR)-major histocompatibility complex 
(MHC) engagement and costimulation, naïve T-cells expand in the lymph nodes without establishing their effector profiles, thereby increasing the precursor frequency to a given antigen. Although initially activated to divide and secrete interleukin (IL)-2, they arrest after 3-4 days of division due to cytotoxic T-lymphocyte antigen-4 (CTLA-4) accumulation in the membrane; an important factor that inhibits cell cycle progression and safeguards against unnecessary T-cell expansion. These cells will eventually leave the lymph nodes and, upon re-exposure to antigen within an inflammatory cytokine-rich site, primed cells would be released of their CTLA-4 hold by activated signal transducer and activator of transcription (STAT)-4 signals, to undergo effector-cell differentiation with an appropriately matched cytokine repertoire. Thus, STAT-4 signals can link expansion of a pre-activated, expanded antigen-specific repertoire with the acquisition of a stabilised cytokine and effector programme. In the absence of STAT signals, activated T-cells remain arrested by CTLA-4 in an anergic state, thus preventing the mischievous development of an unregulated effector response. Indeed, competent signals provided in inappropriately inflamed tissues may release the anergic programme established within pre-activated cell populations and may contribute to the development of autoimmunity [17].

The finding of activated STAT-4 and interferon (IFN)- $\gamma$ in CD4+ T-cells in bronchial biopsies and BAL by DI STEFANO et al. [1] fits with the model described previously. It could work as follows. After antigen presentation by DCs, T-cells start to proliferate and, after a few days in the lymph nodes, they migrate to the inflamed lung, which is the source of antigens. In the lung, other APCs (macrophages and endothelial cells), which are capable of MHC class II presentation and IL-12 production, will engage the TCR, and IL-12 will induce phosporylation of STAT-4, subsequent STAT-4 nuclear translocation and IFN- $\gamma$ gene induction in the T-cells, and, thus, a T-helper (Th)-1 commitment in the T-cells. At the same time, STAT-4 will release the hold on T-cell effector function as a result of CTLA-4 allowing the T-cells to become aggressive effectors with the full potential of causing lung injury [16]. Along these lines, Di STEFANo et al. [1] found that the increasing expansion of activated STAT- 4 and IFN- $\gamma$ in the CD4+ T-cells correlated with decreasing lung function, suggesting that the activated T-cells, through their Th-1 cytokine repertoire and along with the CD8+ T-cells and the innate immune cell brought in by the Th-1 cytokines, are indeed damaging the lung.

It should not come as a surprise that the activated T-cells described by Di STEFANO et al. [1] in the airways and BAL of smokers are CD4+ T-cells, when it has been thought that the main culprit in COPD is the CD8+ T-cell; they both are. To reach full activation as effector cells, CD8+ T-cells need stimulatory signals from CD4+ T-cells at the time of antigen presentation; hence, the same $\mathrm{DC}$ has to present the same antigen to CD4+ and CD8+ T-cells in order to activate the CD8+ T-cells [18]. This is possible by cross-presentation of the antigens arising in the inflamed tissue by either heat shock proteins or phagosomes to neighbouring DCs. These antigens are then processed by the DCs, mainly through the nonacidic proteasome pathway, loaded to the MHC class I and presented to the CD8+ T-cells. A relatively small proportion of the antigen enters an acidic compartment and is loaded into the MHC class II molecules, leading to stimulation of CD4+ T-cells, and allowing for the simultaneous activation of CD4+ and CD8+ cells [19].

Since CD4+ T-cells are also activated, they will migrate to the inflamed tissue: the source of antigens, which is the lung in this case. Thus, the T-cell infiltration in COPD is composed of CD4+ and CD8+ T-cells [2], which is as it should be. It is not surprising that CD8+ T-cells are more abundant, since after activation the clonal expansion of CD8+ T-cells are orders of magnitude larger than CD4+ clonal expansion.

Thus, Di STEFANO et al. [1] bring an important addition to the hypothesis that COPD may be caused by T-cell inflammation and autoimmunity. As only antigens can induce T-cell activation and expansion, and only primed and activated T-cells migrate and enter inflamed tissues (naïve T-cells cannot), the CD8+ and CD4+ T-cells in the lung must be activated. The finding of activated STAT- 4 and IFN- $\gamma$ in the lung T-cells is an important piece of evidence towards this contention. Furthermore, the activating signals arising in the lung tissue have to be competent enough [17] to overcome the hold exerted by CTLA-4, an important protector of untoward T-cell expansion, activation and potential progression to autoimmunity [20].

It seems that the mounting evidence implicating the T-cells as an important component of the inflammation and the disease in smokers is overwhelming. If we accept that the T-cells are part of the inflammatory component, we have to accept the reason why $\mathrm{T}$-cells are in the lung, i.e. they are responding to an antigen challenge originating in the lung. If this is the case, I do not think that it is possible to escape the conclusion that COPD is a disease produced, at least in part, by antigens (self or modified-self) from the lung (autoimmune) secondary to smoking, as has been suggested previously [2-4]. Of course, none of this could be possible without a significant and persistent innate immune inflammation, comprising neutrophils and macrophages.

It would be important to accept or at least explore this possibility since it might lead us to a better understanding and, thus, new and perhaps more effective therapeutic approaches to the disease.

\section{References}

1. Di Stefano A, Caramori G, Capelli A, et al. STAT4 activation in smokers and patients with chronic obstructive pulmonary disease. Eur Respir $J$ 2004; 24: 78-85.

2. Majo J, Ghezzo H, Cosio MG. Lymphocyte population and apoptosis in the lungs of smokers and their relation to emphysema. Eur Respir J 2001; 17: 946-953.

3. Cosio MG, Majo J, Cosio MG. Inflammation of the airways and lung parenchyma in COPD: role of T cells. Chest 2002; 121: Suppl. 5, 160S-165S.

4. Agusti A, MacNee W, Donaldson K, Cosio MG. Hypothesis: does COPD have an autoimmune component? Thorax 2003; 58: 832-834.

5. Rose N, Afanasyeva M. Autoimmunity: busting the atherosclerotic plaque. Nat Med 2003; 9: 641-642.

6. Stemme S, Faber B, Holm J, Wiklund O, Witztum JL, Hansson GK. T lymphocytes from human atherosclerotic plaques recognize oxidized low density lipoprotein. Proc Natl Acad Sci USA 1995; 92: 3893-3897.

7. Finkelstein R, Fraser RS, Ghezzo H, Cosio MG. Alveolar inflammation and its relation to emphysema in smokers. $\mathrm{Am}$ J Respir Crit Care Med 1995; 152: 1666-1672.

8. Butcher EC, Picker LJ. Lymphocyte homing and homeostasis. Science 1996; 272: 60-66.

9. Hunninghake GW, Crystal RG. Cigarette smoking and lung destruction. Accumulation of neutrophils in the lungs of cigarette smokers. Am Rev Respir Dis 1983; 128: 833-838.

10. Martin TR, Raghu G, Maunder RJ, Springmeyer SC. The effects of chronic bronchitis and chronic air-flow obstruction on lung cell populations recovered by bronchoalveolar lavage. Am Rev Respir Dis 1985; 132: 254-260.

11. Matzinger P. The danger model: a renewed sense of self. Science 2002; 296: 301-305. 
12. Abbas AK, Lichtman AH, Pober JS. Cellular and molecular immunology. Saunders Text and Review Series. 4th Edn. New York, W.B. Saunders, 2000; pp. 291-308.

13. Mills PR, Davies RJ, Devalia JL. Airway epithelial cells, cytokines, and pollutants. Am J Respir Crit Care Med 1999; 160: S38-S43.

14. Rose NR. The role of infection in the pathogenesis of autoimmune disease. Semin Immunol 1998; 10: 1-100.

15. Rao T, Richardson B. Environmentally induced autoimmune disease: potential mechanisms. Environ Health Perspect 1999; 107: Suppl. 5, 737-742.

16. Mohrs M, Lacy D, Locksley M. Stat signal release activated naïve Th cells from an anergic checkpoint. J Immunol 2003; 170: $1870-1876$.
17. Luhder F, Chambers C, Allison JP, Benoist C, Mathis D. Pinpointing when $\mathrm{T}$ cell costimulatory receptor CTLA-4 must be engaged to dampen diabetogenic T cells. Proc Natl Acad Sci USA 2000; 97: 12204-12209.

18. Ridge JP, Di Rosa F, Matzinger P. A conditioned dendritic cell can be a temporal bridge between a CD4+ T-helper and a T-killer cell. Nature 1998; 393: 474478.

19. Srivastava P. Interaction of heat shock proteins with peptides and antigen presenting cells: chaperoning of the innate and adaptive immune responses. Annu Rev Immunol 2002; 20: 395-425.

20. Ueda H, Howson JM, Esposito L, et al. Association of the Tcell regulatory gene CTLA-4 with susceptibility to autoimmune disease. Nature 2003; 423: 506-511. 